\title{
EL TRATAMIENTO DE LOS BENEFICIOS EMPRESARIALES SEGÚN EL MODELO DE CONVENIO OCDE 2010 Y SU REPERCUSIÓN PARA LA RED CHILENA DE CONVENIOS DE DOBLE IMPOSICIÓN*
}

\section{RODOLFO SALASSA BOIX**}

RESUMEN: En virtud de la trascendencia y el alcance que tiene la modificación del artículo 7 del Modelo de Convenio de la OCDE 2010 y de la influencia que este Modelo implica para la red chilena de convenios de doble imposición, el trabajo se enfoca en dos grandes objetivos: contrastar la nueva versión del mentado artículo con su redacción anterior (Modelo de Convenio de la OCDE 2008) y analizar la repercusión que tuvo, y debería tener, la reforma de dicho precepto para los convenios fiscales chilenos. Luego de un minucioso repaso de los convenios de doble imposición firmados por Chile, nuestro análisis refleja que ninguno se adapta a la nueva versión del artículo 7 , esto incluye a los acuerdos vigentes al momento de la reforma de 2010 y los firmados por posterioridad a esta. Entendemos que a los fines de mantener una línea coherente con su historia en materia de convenios fiscales internacionales, sumado a su no muy lejana incorporación como miembro de la OCDE (2010), sería conveniente que la Administración chilena siga, al menos en los nuevos tratados, los recientes lineamientos de la OCDE.

PALABRAS CLAVE: Doble imposición internacional - Modelo de convenio de la OCDE 2010 - Beneficios empresariales - Planificación tributaria internacional - Convenios de doble imposición.

Fecha de recepción: 14 de agosto de 2013.

Fecha de aceptación: 3 de diciembre de 1013.

** Licenciado en Derecho. Magíster en Derecho de la Empresa y la Contratación. Doctor en Derecho (mención de calidad europea). Profesor de Derecho Tributario y Financiero de la Universidad Rovira i Virgili de Tarragona, España (grado y postgrado). Inclusión en la publicación bibliográfica "Marquis who's who in the World 2012", New Jersey, 2012. Investigador en el Proyecto de Investigación y Desarrollo titulado: "Análisis jurídico-financiero de la inversión empresarial española en Asia y Latinoamérica". Universidad Rovira i Virgili (2011-2013). Referencia: DER2010-15507. Correo electrónico: rodolfoboix@hotmail.com 


\title{
THE FISCAL TREATMENT OF PROFITS ACCORDING TO THE 2010 OECD CONVENTION MODEL AND ITS IMPACT ON THE CHILEAN NETWORK OF DOUBLE TAXATION AGREEMENTS
}

\begin{abstract}
Considering the significance and scope of the Article 7 amendment of the 2010 OECD Convention Model and the influence that this model implies for the Chilean network of double taxation convention, the paper focuses on two main objectives: contrast the new version of the aforementioned Article with its previous 2008 version and analyze the impact that this provision had, and should have, for Chilean tax treaties. After a thorough review of double taxation agreements signed by Chile our analysis shows that none is adapted to the new version of Article 7, this includes existing agreements at the time of the 2010 reform and subsequent treatments signed after that date. We understand that in order to maintain a consistent line with its international tax treaties history, and considering its recent incorporation as member of the OECD (2010), it is desirable that Chilean Administration should follow, at least in the new treaties, these recent OECD guidelines.
\end{abstract}

KEY WORDS: International double taxation - 2010 OECD Convention model - Profits - International tax planning - Double tax convention

Sumario: 1) Introducción. 2) La reforma del artículo 7 MCOCDE 2010 y su comparación con el artículo 7 del MCOCDE 2008. 2.1) Contextualización. 2.2) Distribución de la potestad tributaria. Regla general. 2.3) Imputación de la renta a los establecimientos permanentes. 2.4) Criterios para la determinación de las utilidades de los establecimientos permanentes. (2.4.1) La determinación de las utilidades de los establecimientos permanentes MCOCDE 2008. (2.4.2). La determinación de las utilidades de los establecimientos permanentes MCOCDE 2010. 2.5) Métodos para evitar la doble imposición internacional. 3) Influencia del MCOCDE en la red chilena de CDI. 4) Repercusiones del nuevo artículo 7 en la red de CDI de Chile. 5) Conclusiones. 6) Bibliografia.

\section{1) INTRODUCCIÓN}

Es sabido que el Comité de Asuntos Fiscales de la OCDE revisa regularmente el contenido de su modelo de convenio, lo cual incluye no solo a sus artículos sino también a los comentarios sobre estos. La última revisión del Modelo de Convenio de la OCDE (MCOCDE) se produjo 
en el año 2010 y contiene modificaciones sobre el articulado y ciertos comentarios de la versión anterior, que databa del año 2008. El día 21 de mayo de 2010 el Comité dio a conocer el borrador ${ }^{1}$ con las actualizaciones del MCOCDE 2010 y el 22 de julio de ese mismo año se publicaron las actualizaciones definitivas del MCOCDE $2010^{2}$. Finalmente, el 13 de septiembre de 2010 se hizo público el texto consolidado y definitivo del nuevo MCOCDE acorde a su nueva versión.

Dentro de las modificaciones realizadas por el nuevo MCOCDE se encuentra el texto de solo un artículo (art. 7), referido a los beneficios (o utilidades) empresariales, y los comentarios de varios artículos (arts. 1, 4, $5,7,8,10,11,12,13,15,21,22,23 \mathrm{~A}, 23 \mathrm{~B}, 24,25$ y 26). A juzgar por estas revisiones se advierte que uno de los temas centrales de la reforma fue la distribución de competencias fiscales a la hora de gravar los beneficios empresariales, máxime si tenemos en cuenta que la mayor cantidad de comentarios retocados fueron justamente los correspondientes al artículo 7.

En virtud de la trascendencia y el alcance que tiene la modificación del artículo 7 del MCOCDE 2010 y de la influencia que este modelo implica para la red chilena de convenios de doble imposición, el trabajo se enfoca en dos grandes objetivos: contrastar la nueva versión del mentado artículo con su redacción anterior (MCOCDE 2008) y analizar la repercusión que tuvo, y las que debería tener, la reforma de dicho precepto para los convenios fiscales chilenos. Para ello dividimos la producción en tres partes, las cuales entendemos necesarias y conducentes para alcanzar el objetivo planteado. En primer lugar, repasaremos brevemente en qué consiste la reforma del artículo 7 del MCOCDE 2010 en comparación con su versión de 2008. En segundo lugar, analizaremos hasta qué punto el MCOCDE es relevante para la configuración de los CDI celebrados por Chile en cuanto a la regulación de los beneficios empresariales; ello nos obliga a revisar no solo el MCOCDE sino también el Modelo de Convenio de la ONU (MCONU), atento que son los dos grandes modelos de convenio de doble imposición que siguen la gran mayoría de países. En tercer lugar, nos centraremos de lleno en las implicaciones concretas que la reforma del artículo 7 del MCOCDE 2010 conlleva para la red chilena de CDI evaluando los acuerdos que finalmente adoptaron tales modificaciones. Para cerrar el trabajo se exponen las conclusiones finales.

Este estudio nos permitirá conocer cuáles son las alternativas que tuvo (y tiene) la Administración chilena ante la modificación del tratamiento de los beneficios empresariales en el MCOCDE 2010; comprobar cuál fue la opción elegida hasta el momento en los CDI que sucedieron 
dicha reforma y, finalmente, proponer, según nuestro criterio, cuál es la elección que se habría de adoptar.

\section{2) LA REFORMA DEL ARTíCUlO 7 DEL MCOCDE 2010 Y SU COMPARACIÓN CON EL ARTÍCULO 7 DEL MCOCDE 2008}

\section{1) CONTEXTUALIZACIÓN}

El artículo 7 del MCOCDE, históricamente vinculado a los beneficios empresariales, no ha permanecido inerte con el paso del tiempo sino que, por el contrario, fue objeto de diversas interpretaciones y modificaciones por parte del Comité de Asuntos Fiscales de la OCDE. El criterio fijado en 1977 para determinar las utilidades imputables a los establecimientos permanentes generaba mucha incertidumbre a la hora de llevarlo a la práctica; ello provocó que en el año 1993 el Comité publicara el conocido "Proyecto de atribución de utilidades de los establecimientos permanentes".

Luego de más de una década, concretamente en el año 2008, fue configurada una nueva versión del Proyecto de atribución de utilidades de los establecimientos permanentes. Dicho documento plasmó el principio separate entity approach, por medio del cual se considera al establecimiento permanente como si fuera una entidad separada e independiente de su casa matriz ${ }^{3}$. Como consecuencia de este último Proyecto se propuso un nuevo texto para el Artículo 7. La modificación más significativa consistió en reemplazar el $3^{\text {er }}$ párrafo de la norma para incorporar un mecanismo de ajuste de los ingresos imputables a los establecimientos permanentes (gastos deducibles), considerando a este como una entidad separada e independiente de su casa matriz (arm's length).

Las modificaciones más relevantes al mentado artículo se produjeron en la reforma del MCOCDE 2010, por medio de la cual se trastrocó sensiblemente el texto del artículo de ciernes y buena parte de sus comentarios. De todas formas, la revisión del artículo 7 y sus comentarios no fue la única modificación del nuevo MCOCDE, también se reformaron los comentarios de gran parte de su articulado, los cuales escapan al objeto de estudio del presente trabajo.

Las modificaciones al artículo 7 se realizaron mediante un proceso gradual que se llevó a cabo mediante dos etapas bien diferenciadas:

\footnotetext{
3 Castañeda Ricci, S. \& Muñoz García, L. F. (2010) Algunos comentarios en relación con la propuesta de modificación al artículo 7 del Convenio Modelo de la OCDE, en cuanto a la forma de atribuir utilidades a un establecimiento. México: PricewaterhouseCoopers, p. 1.
} 
Primera etapa: modificación de los comentarios del artículo 7 del MCOCDE 2005 (fue la versión de 2008). Se forjaron interpretaciones que irían abriendo paso a la nueva redacción e interpretación del artículo 7 , aunque sin contradecir las versiones del modelo previas a estas incorporaciones;

Segunda etapa: revisión y actualización del artículo 7 del MCOCDE 2008 y sus comentarios, para concluir la nueva interpretación del artículo en 2010 .

\section{2) DistribUCiÓN DE LA POTESTAD TRIBUTARIA. REGLA GENERAL}

Los beneficios empresariales son regulados por el artículo 7 del MCOCDE 2010. El punto 1 de este artículo, en su primera parte, define en qué medida el Estado donde se encuentra el establecimiento permanente puede gravar las utilidades obtenidas por su casa matriz cuando esta no reside en su territorio.

De esta manera, los beneficios empresariales percibidos en un Estado Contratante solo pueden gravarse en ese Estado, a menos que aquellos se obtengan en otro Estado Contratante por medio de un establecimiento permanente radicado en este último ${ }^{4}$. Esta es la regla general de distribución competencial con la que coinciden los modelos de convenio bajo análisis (2008 y 2010) y, en general, la mayoría de los CDI.

Cuando las rentas se obtienen con la intervención de un establecimiento permanente la competencia tributaria no es exclusiva de un solo Estado sino compartida, de manera que, incluso bajo el amparo de un CDI, aún pueden producirse supuestos de doble imposición. Para neutralizar estos efectos ${ }^{5}$, lógicamente indeseados, habrá que recurrir al artículo 23 de los modelos convenios, también utilizado normalmente en los acuerdos bilaterales.

El fundamento de la regla general antes mencionada gira en torno a la idea internacionalmente aceptada de que una empresa residente en un Estado Contratante, que realiza actividades empresariales en otro Estado Contratante, no participa de forma relevante en la vida económica de este último sino opera en su territorio a través de un establecimiento permanente ${ }^{6}$.

$4 \quad$ Artículo 7.1, primera parte, del MCOCDE 2008 y del MCONU.

5 "Nótese igualmente que el ejercicio de tal poder tributario por el Estado de la fuente genera la obligación de eliminar la doble imposición internacional sobre tales beneficios por parte del Estado de la residencia de la empresa, en tanto cuanto este último Estado contribuya a la generación de tal fenómeno" (Calderón Carrero, J. M. \& Carmona Fernández, N. (2005) Convenios Fiscales Internacionales. Valencia: CISS, p. 127).

6 Calderón Carrero, J. M. (2004): "Comentarios al artículo 7". En Ruiz García, José Ramón \& Calderón Carrero, José Manuel (Coordinadores): Comentarios a los convenios para evitar la doble imposición y prevenir la evasión fiscal concluidos por España (Análisis a la luz del 
Esta regla general tiene sus excepciones ya que la redacción del artículo 7 , en ambos modelos (2008 y 2010), dota al precepto de un carácter subsidiario en el sentido que su contenido se aplica siempre y cuando las rentas no sean subsumibles dentro de alguna de las otras categorías de renta que contemplan otra norma del modelo ${ }^{7}$. Este precepto es el que otorga sustantividad a los demás artículos que también regulan las actividades empresariales (dividendos, intereses, regalías, entre otros) ${ }^{8}$.

\section{3) IMPUTACIÓN DE LA RENTA A LOS ESTABLECIMIENTOS PERMANENTES}

Si bien la redacción de la regla general del MCOCDE 2010 es idéntica a la de su homólogo de 2008 (art. 7.1, 1a parte), no ocurre lo mismo con la sección de la norma que regula la imputación de la renta al establecimiento permanente (art. 7.1, 2a parte). El Modelo de 2008 decía textualmente que "si la empresa realiza o ha realizado su actividad de dicha manera [mediante un establecimiento permanente], las utilidades de la empresa pueden someterse a imposición en el otro Estado, pero solo en la medida en que puedan atribuirse a ese establecimiento permanente". Por su parte, el artículo 7.1, 2a parte, el MCOCDE 2010 establece que "si la empresa realiza su actividad de dicha manera", es decir, por medio de un establecimiento permanente, "los beneficios imputables al establecimiento permanente de conformidad con las disposiciones del apartado 2 pueden someterse a imposición en ese otro Estado"".

La versión de 2010 ya no dice expresamente que las utilidades de la empresa podrán gravarse "...solo en la medida en que puedan atribuirse a ese establecimiento permanente...", lo que podría llevarnos a pensar que el criterio ha cambiado. No obstante, a pesar de esta omisión, la esencia de la disposición permanece intacta ya que en realidad se dice lo mismo pero invirtiendo la frase. De manera que si acomodamos las oraciones del nuevo modelo podemos decir que las utilidades de la empresa podrán gravarse respecto a "...los beneficios imputables al establecimiento permanente de conformidad con las disposiciones del apartado 2...”. Asimismo, y para

modelo de Convenio de la OCDE y de la legislación y la jurisprudencia española). La Coruña: Fundación Pedro Barrié de la Maza-Instituto de Estudios Económicos de Galicia, p. 414. Artículo 7.7 del MCOCDE 2008 y 2010.

8 Reol Jiménez, T. \& Velayos Jiménez, F. (2007) “Utilidades empresariales con y sin establecimiento permanente”. En Cordón Esquerro, T. (Director): Manual de fiscalidad internacional, Vol. I. Madrid: Instituto de Estudios Fiscales, p. 837.

9 Traducción tomada del Modelo de Convenio Tributario sobre la Renta y sobre el Patrimonio de julio de 2010, adaptado por el Instituto de Estudios Fiscales de España. La versión original del artículo 7.1 del MCOCDE 2010 dice lo siguiente: "Profits of an Enterprise of a Contracting State shall be taxable only in that State unless the enterprise carries on a business in the other Contracting State through a permanent establishment situated therein. If the enterprise carries on business as aforesaid, the profits that are attributable to the permanent establishment in accordance with the provisions of paragraph 2 may be taxed in that other State". 
apoyar la postura de la inmutabilidad del criterio, también hay que decir que los comentarios de dicho precepto coinciden en ambos modelos ${ }^{10}$.

En virtud de lo expuesto, el derecho de los Estados Contratantes para gravar la renta de establecimientos permanentes situados en su territorio no se extiende a los beneficios que obtenga la casa central sin la mediación del dicho establecimiento ${ }^{11}$. En definitiva, entendemos que a pesar de las modificaciones en el texto, todo el primer punto del artículo 7 permanece en esencia idéntico en ambos modelos.

\section{4) CRITERIOS PARA LA DETERMINACIÓN DE LAS UTILIDADES DE LOS ESTABLECIMIENTOS PERMANENTES}

\subsection{1.) La determinación de las utilidades de los establecimientos permanentes en el MCOCDE 2008}

La determinación de las utilidades del Modelo de 2008 estaba desarrollada básicamente en los puntos 2 y 3 del artículo 7 . El primero de estos apartados establecía que las utilidades imputables al establecimiento permanente eran las que este hubieran obtenido si, en lugar de tratar con su sede central, lo hubiese hecho con una empresa totalmente independiente y en condiciones y precios normales de mercado. De forma que la

10 "El segundo principio, expresado en la segunda frase del apartado, es que la potestad tributaria del Estado en el que está situado el establecimiento permanente no se extiende a los beneficios que la empresa pueda obtener en ese Estado que no sean atribuibles al establecimiento permanente. Sobre este punto han existido diferencias de opinión, habiendo defendido algunos paises, tiempo atrás, el principio general de "fuerza de atracción", conforme al que las rentas tales como otros beneficios empresariales, dividendos, intereses y regalias, procedentes de fuentes situadas en sus territorios, estaban plenamente comprendidos en sus potestades tributarias si el beneficiario poseía un establecimiento permanente en dicho territorio, aún cuando resultara obvio que la renta no era imputable a ese establecimiento permanente. Si bien algunos convenios bilaterales incluyen una norma antielusión restringida, basada en un criterio limitado de fuerza de atracción que unicamente se aplica a los beneficios empresariales procedentes de actividades similares a las desarrolladas por el establecimiento permanente, la aplicación del principio general de fuerza de atracción antes descrito se considera actualmente obsoleto como práctica internacional en la politica de convenios. El principio actualmente vigente en los convenios se basa en el criterio de que al gravar los beneficios que una empresa extranjera obtiene en un pais determinado, las autoridades fiscales de ese pais deberán considerar separadamente las diversas fuentes de los beneficios que la empresa obtiene en el mismo y aplicar a cada una de esas fuentes el criterio para la determinación de la existencia de un establecimiento permanente, con sujeción a la posible aplicación de otros artículos del Convenio. Esta solución permite simplificar la administración del impuesto y facilitar el cumplimiento con las obligaciones tributarias, y está mejor adaptada al modo en que normalmente se realizan las actividades económicas..." (Párrafo 10 de los comentarios al artículo 7 del MCOCDE 2008 y párrafo 13 de los comentarios al artículo 7 del MCOCDE 2010).

11 Confr. Párrafos 12 y 17 de los comentarios al artículo 7 del MCOCDE 2010. 
atribución de beneficios al establecimiento descansaba sobre el principio de empresa separada e independiente ${ }^{12}$.

Por su parte, el artículo 7.3 del MCOCDE 2008 permitía la deducción de los gastos realizados para los fines de los establecimientos permanentes, incluyendo los gastos de dirección y generales de administración para dichos fines, tanto si se realizaban en el Estado en que se encuentra el establecimiento como en otro sitio. De esta manera, la norma buscaba básicamente aclarar el principio general del $2^{\circ}$ punto del artículo.

Este tercer apartado indicaba que al calcularse las utilidades del establecimiento permanente era posible deducir determinados gastos, mientras que el apartado 2 establecía la manera en que debían determinarse las utilidades determinadas con arreglo a aquel apartado ${ }^{13}$. En virtud de ello, podemos decir que el punto 3 fijaba los gastos que podían deducirse y el punto 2 determinaba los parámetros que debían orientar dicha deducción ${ }^{14}$.

Tal y como expresaban los comentarios del MCOCDE 2008, las utilidades determinadas de esta forma normalmente coincidían con las obtenidas por aplicación de las reglas habituales de la buena práctica contable $^{15}$. De esta manera, las autoridades fiscales debían partir de la contabilidad (separada) del establecimiento permanente para determinar la atribución de beneficios o llevar a cabo un ajuste de ellos ${ }^{16}$. En caso que fuera necesario realizar ajustes, se habían de sustituir los valores facturados por los precios de mercado ordinarios para los productos idénticos o semejantes, suministrados en las mismas o similares condiciones.

Como hemos adelantado, el anterior artículo 7.3 giraba en torno a la delimitación de los gastos deducibles y vino a completar el artículo 7.2. $\mathrm{Su}$ finalidad era garantizar que los emolumentos derivados de la actividad del establecimiento permanente no fueran rechazados porque el gasto había sido desembolsado fuera del territorio del Estado donde se encontraba el establecimiento permanente o porque el gasto no tenía como única finalidad atender las necesidades generadas la actividad del establecimiento permanente. A partir de los comentarios del Modelo, el establecimiento permanente podría deducir dichos gastos pero sin incluir el margen de beneficios ${ }^{17}$, que sí se incluyó para la casa central ${ }^{18}$.

\footnotetext{
12 El establecimiento permanente debe tributar como un centro de imputación de rentas similar al integrado por la formación de unja empresa separada y residente que opera de forma independiente en relación con las operaciones que realice con personas vinculadas, incluyendo a su propia casa central (García Prats, F. A. (1996) El establecimiento permanente. Madrid: Tecnos, p. 308).

13 Párrafo 28 de los comentarios al artículo 7 del MCOCDE 2008.

14 Calderón Carrero (2004) 453 y ss.

15 Párrafo 14 de los comentarios al artículo 7 del MCOCDE 2008.

16 Calderón Carrero (2004) 437.

17 Párrafo 28 de los comentarios al artículo 7 del MCOCDE 2008.

18 En esta línea Calderón Carrero (2004) 466.
} 
Finalmente, dentro de los criterios para determinar las utilidades de los establecimientos permanentes, también queremos destacar el contenido del $5^{\circ}$ párrafo del MCOCDE, que se relaciona directamente con los puntos que venimos comentando, que decía lo siguiente:

"5. No se atribuirán utilidades a un establecimiento permanente por la simple compra de bienes o mercancías para la empresa".

Si bien este párrafo parecía bastante similar al artículo 5.4.d) del mismo Modelo, en el cual se excluían las "oficinas de compras" de la categoría de establecimiento permanente, cada precepto apuntaba a diferentes cuestiones.

El antiguo artículo 7.5 no se refería a las organizaciones establecidas únicamente para realizar compras ya que tales oficinas no eran consideradas como establecimientos permanentes y, por lo tanto, no se le aplicaba este artículo. En realidad hacía mención a los establecimientos que, además de realizar otras actividades empresariales, también efectuaban compras para su sede central. En tal caso, las utilidades de dicho establecimiento no debían incrementarse en un importe estimado al de las utilidades de la actividad compradora ${ }^{19}$.

De todas maneras, y como veremos en el punto siguiente, el criterio del artículo 7.5 del MCOCDE 2008 fue puesto en tela de juicio por la OCDE y así lo ha hecho saber en la última versión de su Modelo (2010).

\subsection{2.) La determinación de las utilidades de los establecimientos permanentes MCOCDE 2010}

En el MCOCDE 2010, la determinación de los beneficios empresariales del establecimiento permanente se encuentra regulada en el $2^{\circ}$ apartado del artículo 7 del MCOCDE 2010, que dice lo siguiente:

"2. A los efectos de este artículo, y del artículo [23 A] [23 B], los beneficios imputables al establecimiento permanente en cada Estado contratante a los que se refiere el apartado 1 son aquellos que el mismo hubiera podido obtener, particularmente en sus operaciones con otras partes de la empresa, si fuera una empresa distinta e independiente que realizase actividades idénticas o similares, en las mismas o análogas condiciones, teniendo en cuenta las funciones realizadas, los activos utilizados y los riesgos asumidos por la empresa a través del establecimiento permanente $y$ de las restantes partes de la empresa" 20 .

19 Párrafo 57 de los comentarios al artículo 7 del MCOCDE.

20 Traducción tomada del Modelo de Convenio Tributario sobre la Renta y sobre el Patrimonio de julio de 2010, adaptado por el Instituto de Estudios Fiscales de España. La version original establece literalmente que "for the purposes of this Article and Article [23A] [23B], the profits are attributable in each Contracting State to the permanent establishment referred 
A partir de este artículo las actividades del establecimiento permanente se identificarán a través de un análisis funcional, que consiste en suponer que el establecimiento es una empresa separada e independiente capaz de llevar a cabo funciones; poseer y utilizar activos, asumir riesgos; y celebrar transacciones con otras empresas, relacionadas y no relacionadas, así como la empresa de la cual forma parte ${ }^{21}$. De manera que en esta nueva versión es primordial tener en consideración las funciones realizadas, los activos utilizados y los riesgos asumidos por las partes.

Esta disposición intenta ser más específica respecto a los elementos a tener en cuenta para aplicar el principio de empresa separada e independiente. De todas maneras, se trata de consideraciones bastante amplias cuya determinación dependerá de cada caso en particular. Cuando se habla de "riesgos", por ejemplo, nos preguntamos a qué riesgos se refiere concretamente el artículo. En principio está claro que toda actividad comercial conlleva una serie de riesgos, con lo cual queda abierta la posibilidad de determinar los riesgos que se tomen en cuenta a la hora de aplicar el mencionado principio.

Por otra parte, según la norma bajo análisis, la determinación de las rentas implica el cálculo de los beneficios (o pérdidas) derivados de toda la actividad, incluyendo las transacciones con partes independientes, las transacciones con partes relacionadas y los acuerdos con las otras partes de la empresa ${ }^{22}$.

Tal y como expresan los comentarios del MCOCDE 2010, la determinación de utilidades del establecimiento permanente del nuevo artículo consta de dos pasos bien diferenciados ${ }^{23}$. El primero consiste en realizar un análisis fáctico-funcional que implica la:

- atribución al establecimiento los derechos y obligaciones derivadas de las operaciones con la casa matriz y/o otras partes del grupo;

to in paragraph 1 are the profits it might be expected to make, in particular in its dealings with other parts of the enterprise, if it were a separate and independent enterprise engaged with other parts of the enterprise, if it were a separate and independent enterprise engaged in the same or similar activities under the same or similar conditions, taking into account the functions performed, assets used and risks assumed by the enterprise through the permanent establishment and through the other parts of the enterprise".

21 En la aplicación del principio arm's length resultarán aplicable los lineamientos sobre precios de transferencia establecidos por la OCDE (OECD Transfer pricing guidelines).

22 Párrafo 20 de los comentarios al artículo 7 del MCOCDE 2010. El tema de las operaciones intragrupo motivaron un gran debate ya que el anterior artículo 7.3 se interpretaba en el sentido de que prohibía el reconocimiento de este tipo de transacciones (Tonino, H. \& Cooper, J. (2010) Princing a hypothetical. OECD Observer, No 276-277, diciembre 2009 enero 2010).

23 Para un análisis más profundo recomendamos consultar Valente, P. (2010) "Attribuzione del reddito alla stabile organizzazione: il Rapporto OCSE del 2010". il Fisco, No 43, 22 de novembre 2010, p. 1-7000. 
- individualización de los activos económicamente poseídos y utilizados por el establecimiento;

- identificación de los riesgos inherentes a la función desarrollada, teniendo en cuenta la eventual transmisión de dichos riesgos o de su gestión a la casa central y/o a otras partes del grupo;

- determinación de otras funciones del establecimiento;

- atribución de fondos (capital libre y pasivo exigible) para determinar intereses deducibles por el establecimiento;

- identificación de la existencia y naturaleza de eventuales acuerdos con la casa matriz y/o las otras partes del grupo.

El segundo paso está dado por la aplicación de los lineamientos generales de la OCDE sobre precios de transferencia a los acuerdos celebrados entre el establecimiento permanente y las otras partes del grupo ${ }^{24}$. Este proceso se realiza a través de la:

- comparación de las operaciones sujetas a los mencionados acuerdos y las operaciones no sujetas a condiciones particulares. Para lo cual se tendrán en cuenta las características de los bienes y servicios, las circunstancias económicas y estratégicas de negocio (factores directos), o los análisis funcionales y términos contractuales (analogía);

- aplicación por analogía de los métodos propuestos por la Guía OCDE sobre Precios de Transferencia para arribar a una compensación independiente de los acuerdos y condiciones espaciales pactadas.

Respecto a la comparación de versiones, el artículo 7 del Modelo 2008 utilizaba cinco párrafos para determinar las utilidades imputables al establecimiento permanente (párr. 2, 3, 4, 5 y 6), aunque nosotros solo analizamos tres de ellos (párr. 2, 3 y 5). Con la nueva redacción de 2010 esta tarea queda únicamente en manos del mencionado artículo 7.2 y sus comentarios.

El MCOCDE 2010 aclara que las condiciones para la deducción de los gastos deben ser determinadas por la normativa doméstica, aunque siempre sujeta a los preceptos del Modelo, en especial los de su artículo $24.3^{25}$. En aras de velar por el principio de no discriminación, esta última norma establece que los gastos realizados a los fines de un establecimiento permanente situado en un Estado Contratante, son deducibles bajo las

24 Párrafo 23 de los comentarios al artículo 7 del MCOCDE 2010.

25 Párrafo 30 de los comentarios al artículo 7 del MCOCDE 2010. Asimismo, el párrafo 66 de los comentarios al mismo artículo establece que el artículo 7 tiene como objetivo "la localización de la potestad tributaria entre los Estados Contratantes. Dicha norma solo sirve para localizar las ganancias y pérdidas a efectos de distribuir la potestad tributaria y no se refiere a la cuestión sobre cuáles ganancias son gravadas y cuáles pérdidas son deducibles, ya que esto es materia del derecho interno". 
mismas condiciones que los gastos efectuados a los fines de una empresa radicada en dicho Estado ${ }^{26}$.

Finalmente cabe destacar que, a diferencia de la versión anterior, ahora se atribuyen al establecimiento permanente las utilidades derivadas de la mera compra de bienes o mercancías para la empresa. Recordemos que el artículo 7.5 del Modelo 2008 permitía excluir este tipo de rentas pero el Comité de Asuntos Fiscales consideró que no era acorde al principio arm's length dejar fuera estos beneficios por el solo hecho de que se trate de compras para la empresa ${ }^{27}$.

\section{5) MÉTODOS PARA EVITAR LA DOBLE IMPOSICIÓN INTERNACIONAL}

A diferencia de su predecesor, el $3^{\text {er }}$ punto del artículo 7 del MCOCDE 2010 ya no habla de los gastos deducibles sino que incorpora un método de compensación y ajustes para evitar la doble imposición de las utilidades. Es un mecanismo muy similar al del artículo 9.2 del MCOCDE (idéntico en ambos modelos) ${ }^{28}$.

La aplicación del artículo 7.2 puede generar diferencias entre los Estados Contratantes, es por ello que el Modelo incorpora un mecanismo para resolver estas posibles discrepancias y eliminar así las eventuales situaciones de doble imposición. En virtud de ello, el artículo 7.3, en combinación con el artículo 23 también del MCOCDE 2010, pretende asegurar que no se produzca una doble imputación sobre los beneficios atribuibles al establecimiento permanente ${ }^{29}$. Concretamente, el párrafo 3 del nuevo artículo 7 dice lo siguiente:

"3. Cuando de conformidad con el apartado 2 un Estado contratante ajuste los beneficios imputables al establecimiento permanente de una empresa de uno de los Estados Contratantes y, en consecuencia, grave los beneficios de la empresa que han sido gravados por el otro Estado, ese otro Estado, en la medida en que sea necesario para eliminar la doble imposición sobre dichos beneficios, practicará el ajuste correspondiente en la cuantía del impuesto aplicado sobre los mismos. En caso necesario, las autoridades competentes de los Estados contratantes se consultarán para la determinación de dicho ajuste" 30 .

26 Párrafos 34 de los comentarios al artículo 7 y 40 del artículo 24 del MCOCDE 2010.

27 Párrafo 43 de los comentarios al artículo 7 del MCOCDE 2010.

28 El propio Modelo, en el párrafo 58 de los comentarios a su artículo 7 , confirma que ambas disposiciones comparten los mismos caracteres: se aplican por cada Estado en relación a un ajuste hecho en el otro Estado Contratante; deben aplicarse de manera recíproca; no se aplicarán a menos que haya habido un ajuste por parte de alguno de los Estados Contratantes.

29 Párrafo 44 de los comentarios al artículo 7 del MCOCDE 2010.

30 Traducción tomada del Modelo de Convenio Tributario sobre la Renta y sobre el Patrimonio de julio de 2010, adaptado por el Instituto de Estudios Fiscales de España. En inglés, el artículo dice textualmente lo siguiente: "Where, in accordance with paragraph 2, a 
Tal y como aclaran los comentarios, en principio no debería ser necesario recurrir al párrafo 3 . En efecto, la posibilidad de que se genere una doble imposición no se producirá con demasiada frecuencia porque los contribuyentes determinarán la renta del establecimiento de la misma forma en cada Estado Contratante y con arreglo a los criterios del párrafo 2 (arm's length) ${ }^{31}$. Al existir una absoluta coherencia, no solo en el sistema utilizado en cada Estado sino también en los postulados convencionales, no será preciso, prima facie, realizar ningún tipo de ajuste.

El Modelo deja bien en claro que su nueva versión no pretende limitar en lo más mínimo los remedios legales de los contribuyentes para asegurar el cumplimiento de sus disposiciones ${ }^{32}$. De forma tal que si el Estado donde se encuentra el establecimiento permanente no sigue las pautas del artículo 7.2, el contribuyente podrá acudir a los remedios legales domésticos y a los acuerdos amistosos contemplados en el artículo 25 del MCOCDE 2010. Lo mismo ocurrirá si el otro Estado Contratante, en relación con el mentado artículo 23, no calculase los beneficios según las reglas del artículo 7.2 del Modelo de 2010.

Pero si es el contribuyente el que no determina las utilidades del establecimiento acorde a las disposiciones del Modelo, cada Estado estará habilitado para realizar los ajustes necesarios en aras de cumplimentar con el mentado artículo 7.2. Al igual que ocurre con el artículo 9.2, el MCOCDE 2010 no especifica ni el método ni el plazo para realizar dichos ajustes.

A continuación ilustraremos la disposición de este apartado del artículo 7 con un ejemplo propuesto en los comentarios de la última versión del Modelo ${ }^{33}$. Comencemos el supuesto con la siguiente hipótesis: una empresa del Estado R (Estado de la residencia) ha transferido bienes a un establecimiento permanente situado en el Estado F (Estado de la fuente).

La documentación de la empresa refleja que para la determinación de los beneficios del establecimiento las partes acordaron un precio de 90. Supongamos que el Estado F acepta el precio establecido por el contribuyente pero el Estado $\mathrm{R}$ no lo considera ajustado al artículo 7.2 del MCOCDE y entiende que, en aplicación del principio de empresa separada e independiente, debió utilizarse un precio de 110. Bajo estas circunstancias el precio es diferente para ambos países, de manera que la

Contracting State adjusts the profits that are attributable to a permanent establishment of an Enterprise of one the Contracting States and taxes accordingly profits of the Enterprise that have been charged to tax the other State, the other State shall, to the extent necessary to eliminate double taxation on these profits, make an appropriate adjustment to the amount of the tax charged on those profits. In determining such adjustment, the competent authorities of the Contracting States shall if necessary consult each other".

31 Párrafos 46 y 50 de los comentarios al artículo 7 del MCOCDE 2010.

32 Párrafo 52 del los comentarios al artículo 7 del MCOCDE 2010.

33 Párrafos 55 y 56 de los comentarios al artículo 7 del MCOCDE 2010. 
cantidad de 20 puede ser sometida a doble tributación. Aquí se presentan dos posibles opciones:

- que el Estado F acepte que el ajuste realizado por el Estado R está de acuerdo con el párrafo 2, en cuyo caso el párrafo 3 solicitará al Estado $\mathrm{F}$ a que reajuste el tributo según el nuevo precio, es decir 110; o

- que el Estado F no esté de acuerdo el ajuste propuesto por el Estado $\mathrm{R}$, y decida no realizar ningún tipo de reajuste. En dicho caso, la cuestión se resolverá con el procedimiento amistoso de resolución de conflictos del artículo 25.1 del MCOCDE.

\section{3) INFLUENCIA DEL MCOCDE EN LA RED CHILENA DE CDI}

La mayor o menor relevancia que las modificaciones del artículo 7 del MCOCDE 2010 pueda tener para los tratados chilenos va a depender de la mayor o menor influencia que los lineamientos de la OCDE tengan en la red de CDI de Chile. Es por ello que en este acápite nos centraremos en dilucidar cuál es el modelo de convenio más determinante para la celebración de los CDI vigentes, o suscritos pero aún no vigentes, por el gobierno chileno. Siempre enfocados en la regulación de los beneficios empresariales (art. 7).

Históricamente los modelos de convenios internacionales que han guiado la mayoría de acuerdos bilaterales han sido el MCOCDE y el Modelo de Convenio de la ONU (MCONU). El MCOCDE es un documento elaborado por los Estados más desarrollados en la segunda mitad del siglo pasado ante el constante incremento de las transacciones internacionales susceptibles de tributación. Su finalidad era alcanzar una coordinación a nivel mundial que les permitiese asegurar las mismas condiciones fiscales de competencia internacional. Por su parte, el MCONU nació en 1980 en el seno de la ONU para equilibrar las relaciones entre los países desarrollados y los que estaban en vías de desarrollo, ya que los primeros, aprovechándose de su poderío económico, venían imponiendo a los segundos sus condiciones como países exportadores de capital y tecnología. Es por ello que, a diferencia del MCONU, el MCOCDE normalmente intentará que las rentas sean sometidas a tributación en el país de la residencia y no en el de la fuente. Esta circunstancia hace que el MCOCDE normalmente vaya un paso más adelante que el MCONU y que sea el primero en regular las distintas cuestiones de doble imposición internacional; por su parte, el MCONU se dedica a ejercer una función de contrapeso para que los CDI sean más equilibrados, más allá del nivel económico de los Estados firmantes.

Recordemos que estos modelos no definen cómo se han de gravar los beneficios empresariales sino que, por el contrario, se limitan a distribuir 
la potestad tributaria de los Estados contratantes a los fines de neutralizar los efectos perjudiciales de la doble imposición internacional.

A continuación transcribimos el artículo 7 del MOCDE (versión de 2008), a los fines de compararlo con la norma de los convenios chilenos que trata los beneficios empresariales. Atento que el artículo 7 del MCOCDE 2008 es la versión inmediata anterior a la del año 2010, la comparación se realiza según el texto de 2008 y dejamos para el punto 4) de este trabajo el análisis de la nueva versión (2010) y su recepción normativa en los CDI chilenos posteriores a 2010.

"1. Las utilidades de una empresa de un Estado Contratante solamente pueden someterse a imposición en ese Estado, a no ser que la empresa realice su actividad en el otro Estado Contratante por medio de un establecimiento permanente situado en él. Si la empresa realiza o ha realizado su actividad de dicha manera, las utilidades de la empresa pueden someterse a imposición en el otro Estado, pero solo en la medida en que puedan atribuirse a ese establecimiento permanente.

2. Sujeto a lo previsto en el párrafo 3, cuando una empresa de un Estado Contratante realice su actividad en el otro Estado Contratante por medio de un establecimiento permanente situado en él, en cada Estado Contratante se atribuirán a dicho establecimiento las utilidades que este hubiera podido obtener de ser una empresa distinta y separada que realizase las mismas o similares actividades, en las mismas o similares condiciones y tratase con total independencia.

3. Para la determinación de la utilidad del establecimiento permanente se permitirá la deducción de los gastos incurridos para los fines del establecimiento permanente, comprendidos los gastos de dirección y generales de administración para los mismos fines, tanto si se efectuan en el Estado en que se encuentre el establecimiento permanente como en otra parte.

4. Mientras sea usual en un Estado Contratante determinar las utilidades imputables a un establecimiento permanente sobre la base de un reparto de las utilidades totales de la empresa entre sus diversas partes, lo establecido en el párrafo 2 no impedirá que ese Estado Contratante determine de esta manera las utilidades imponibles; sin embargo, el método de reparto adoptado habrá de ser tal que el resultado obtenido sea conforme a los principios contenidos en este artículo.

5. No se atribuirá ninguna utilidad a un establecimiento permanente por el mero hecho de que este compre bienes o mercancias para la empresa.

6. A efectos de los párrafos anteriores, las utilidades imputables al establecimiento permanente se calcularán cada año por el mismo método, a no ser que existan motivos válidos y suficientes para proceder de otra forma.

7. Cuando las utilidades comprendan elementos de rentas regulados separadamente en otros artículos de este Convenio, las disposiciones de aquellos no quedarán afectadas por las de este artículo" (Art. 7, MCOCDE 2008). 
A continuación ofrecemos un breve repaso del esquema general utilizado por los CDI celebrados por Chile a la hora de regular la distribución competencial en cuanto a las utilidades empresariales ${ }^{34}$. A tales fines, agrupamos los acuerdos en cuatro grupos: a) convenios que transcriben literalmente el artículo 7 del MCOCDE; b) convenios que siguen el artículo 7 del MCOCDE pero con algunas omisiones; c) convenios que siguen el artículo 7 del MCOCDE pero con algunos agregados; d) convenios que siguen el artículo 7 del MCOCDE pero con omisiones y agregados; e) convenios que se apartan del MCOCDE para seguir el artículo 7 del MCONU.

\section{a) Convenios que transcriben literalmente el artículo 7 del MCOCDE}

A la hora de regular los beneficios empresariales, los CDI que Chile ha suscrito con los siguientes países siguen al pie de la letra el artículo 7 del MCOCDE 2008: Austria (aún no entró en vigor), Bélgica, Colombia, Croacia, Dinamarca, Ecuador, Irlanda, Malasia, Noruega, Paraguay, Perú, Polonia, Portugal, Rusia, Sudáfrica (aún no entró en vigor), Suiza y Tailandia.

\section{b) Convenios que siguen el artículo 7 del MCOCDE pero con algunas omisiones}

El artículo 7 de los acuerdos bilaterales suscritos con los siguientes países siguen en gran medida el artículo 7 del MCOCDE 2008, aunque omiten incorporar el punto 4 de dicha norma: Corea, España, Nueva Zelanda y Suecia.

El CDI suscrito con Brasil, en lo que a utilidades se refiere, sigue en gran medida el artículo 7 del MCOCDE 2008, con la única diferencia que se prescinde de los apartados 4 y 6 de dicha norma.

\section{c) Convenios que siguen el artículo 7 del MCOCDE pero con algunos agregados}

La regulación de los beneficios empresariales de los Convenios firmados con Francia y Reino Unido también se basa en el mentado artículo 7 del MCOCDE 2008, aunque se agregan algunos puntos especiales que seguidamente veremos.

El Convenio con Francia incorpora en el punto 8 del artículo 7 lo siguiente: "La renta o ganancia atribuible a un establecimiento permanente durante su existencia es imponible en el Estado Contratante donde tal estable-

\footnotetext{
34 La información sobre los CDI suscritos por Chile es recolectada de la página oficial de Servicios de Impuestos Internos de Chile (SII), a fecha de 22 de agosto de 2013, a partir del siguiente enlace: http://www.sii.cl/pagina/jurisprudencia/convenios.htm
} 
cimiento permanente esté situado, aunque los pagos sean diferidos hasta que ese establecimiento permanente haya dejado de existir".

Por su parte, el CDI celebrado Reino Unido ańade tres puntos extras en la parte final del artículo, a saber:

"7. Para la aplicación de los párrafos 1 y 2 de este artículo, las rentas $o$ beneficios que sean imputables a un establecimiento permanente, pueden, no obstante que el establecimiento permanente haya dejado de existir, ser gravadas en el Estado Contratante en el cual estuvo situado.

8. Nada en este Convenio afectará la imposición en Chile de un residente en el Reino Unido en relación a los beneficios atribuibles a un establecimiento permanente en Chile, tanto respecto del impuesto de primera categoría como del impuesto adicional.

9. Nada en este Convenio afectará la aplicación de las disposiciones vigentes del Decreto Ley $N^{\circ} 600$ de la legislación chilena (Estatuto de la Inversión Extranjera) conforme estén en vigor a la fecha de la firma de este Convenio y aún cuando fueren eventualmente modificadas sin alterar su principio general'.

\section{d) Convenios que siguen el artículo 7 del MCOCDE pero con algunas omisiones y agregados}

Dentro de este punto se encuentran los acuerdos suscritos con Australia y Estados Unidos, este último aún no ha entrado en vigor.

El artículo 7 del Acuerdo con Australia sigue gran parte el artículo 7 del MCODE 2008 aunque se omite la incorporación de los párrafos 4 y 6 de este último y se ańaden una serie de puntos especiales que transcribimos a continuación:

"6. No obstante las disposiciones anteriores de este Articulo, las primas respecto de pólizas de seguros emitidas por una empresa de un Estado Contratante pueden someterse a imposición en el otro Estado de conformidad con su Derecho interno. Sin embargo, salvo cuando la prima sea atribuible a un establecimiento permanente de la empresa situado en ese otro Estado, el impuesto aplicado no podrá exceder: a) 5 por ciento del monto bruto de la prima en el caso de pólizas de reaseguros; b) 10 por ciento del monto bruto de las primas en el caso de todas las demás pólizas de seguro.

7. Cuando:

a) un residente de un Estado Contratante tenga derecho como beneficiario efectivo, ya sea directamente o a través de uno o más 'trust estates', a una participación en las utilidades de una empresa explotada en el otro Estado Contratante por el 'trustee' de un 'trust estate' distinto de un 'trust estate' que sea tratado como sociedad para fines impositivos; $y$

b) respecto de esa empresa, ese 'trustee' tendria, conforme a los principios del Artículo 5, un establecimiento permanente en ese otro Estado, la empresa explotada por el 'trustee' se considerará explotada en el otro Estado por aquel residente mediante un establecimiento permanente situado en 
el y esa participación en las utilidades empresariales se atribuirá a ese establecimiento permanente.

8. Ningún ajuste se realizará por un Estado Contratante a las utilidades atribuibles en un año tributario a un establecimiento permanente de una empresa después de la expiración de siete años contados desde la fecha en la cual la empresa ha completado los requisitos de declaración de impuestos de ese Estado para ese año tributario. La disposiciones de este párrafo no se aplicarán en el caso de fraude, negligencia grave o incumplimiento intencional, o cuando, dentro de ese periodo de siete años, haya sido iniciada una auditoría a las utilidades de la empresa por parte de cualquiera de los Estados.

9. Nada en este Convenio afectará la imposición en Chile de un residente de Australia, en relación a las utilidades atribuibles a un establecimiento permanente o una base fija, situados en Chile, tanto respecto del Impuesto de Primera Categoría como del Impuesto Adicional, en la medida en que el Impuesto de Primera Categoría sea completamente deducible en el cálculo del monto del Impuesto Adicional”.

El artículo 7 del Convenio con Estados Unidos también se basa en líneas generales en el artículo 7 del MCOCDE 2008, aunque no se agrega el punto 4 del Modelo y se añaden tres apartados particulares:

"7. Cualquier renta o ganancia atribuible a un establecimiento permanente o base fija durante su existencia puede someterse a imposición en el Estado contratante donde esté situado dicho establecimiento permanente o base fija, aunque los pagos sean diferidos para después que dicho establecimiento permanente o base fija haya dejado de existir.

8. No obstante, lo dispuesto en el párrafo 1, en la ausencia de un establecimiento permanente, Estados Unidos podrá aplicar su impuesto indirecto sobre primas de seguros pagadas a aseguradoras extranjeras y Chile podrá aplicar su impuesto sobre primas de seguros contratados con compañias no establecidas en Chile. Sin embargo y no obstante lo dispuesto en el Artículo 2 (Impuestos Comprendidos), el impuesto no podrá exceder de: a) 2 por ciento del importe bruto de las primas en el caso de pólizas de reaseguro; y b) 5 por ciento del importe bruto de las primas en el caso de cualquier otro tipo de pólizas de seguro.

9. A los efectos del Convenio, el término 'utilidades empresariales' significa la renta obtenida de cualquier comercio o negocio".

\section{e) Convenios que se apartan del MCOCDE para seguir el artículo 7 del MCONU}

A la hora de regular las utilidades empresariales, los CDI suscritos con Canadá y México se vuelcan primordialmente al texto del artículo 7 del MCONU. 
En el primer caso esta influencia se ve fundamentalmente en el punto 3 del artículo que dice lo siguiente:

"Para la determinación de los beneficios del establecimiento permanente se permitirá la deducción de los gastos en que se haya incurrido para la realización de los fines del establecimiento permanente, comprendidos los gastos de dirección y generales de administración, tanto en el Estado en que se encuentre el establecimiento permanente como en otra parte. Sin embargo, no serán deducibles los pagos que efectúe, en su caso, el establecimiento permanente (que no sean los hechos por concepto de reembolso de gastos efectivos) a la oficina central de la empresa o a alguna de sus otras oficinas, a titulo de regalias, honorarios o pagos análogos a cambio del derecho de utilizar patentes, 'know-how' u otros derechos, o a titulo de comisión u otros cargos, por servicios concretos prestados o por gestión $o$, salvo en el caso de un banco, a título de intereses sobre el dinero prestado al establecimiento permanente".

El Convenio con México refleja su acercamiento al MCONU esencialmente en su artículo 7.1 que reza lo siguiente:

"1. Los beneficios de una empresa de un Estado Contratante solamente pueden someterse a imposición en ese Estado, a no ser que la empresa realice su actividad en el otro Estado Contratante por medio de un establecimiento permanente situado en él. Si la empresa realiza o ha realizado su actividad de dicha manera, los beneficios de la empresa pueden someterse a imposición en el otro Estado, pero solo en la medida en que puedan atribuirse a: a) ese establecimiento permanente; b) enajenaciones de bienes o mercancías en ese otro Estado de tipo idéntico o similar a los bienes o mercancías enajenados por medio del establecimiento permanente. Sin embargo, los beneficios derivados de las enajenaciones descritas en la letra b) no serán sometidos a imposición en el otro Estado si la empresa demuestra que dichas ventas han sido realizadas de esa manera por razones distintas a las de obtener un beneficio del presente Convenio".

En definitiva, a partir de este breve pero completo repaso por el artículo 7 de los CDI suscritos por Chile, podemos decir que el MCOCDE ejerce una importante influencia en su normativa convencional, por lo menos en lo que al tratamiento de los beneficios empresariales se refiere. En consecuencia, la nueva versión del artículo 7 del MCOCDE 2010 no puede resultar indiferente para la red de convenios de dicho país. En el punto siguiente veremos en qué medida influye, o podría haber influido, dicho artículo en los CDI chilenos. 


\section{4) REPERCUSIONES DEL NUEVO ARTÍCULO 7 PARA LA RED DE CDI DE CHILE}

A los fines de desarrollar este acápite nos detendremos, por un lado, en la naturaleza jurídica de los modelos de convenios y su relación con los CDI en general y, por el otro, en la postura que viene adoptando la Administración chilena en sus acuerdos bilaterales respecto a la adopción del artículo 7 del MCOCDE 2010.

En relación al primer punto es importante recordar que el Derecho puede clasificarse en hard law (derecho duro) y soft law (derecho blando) según el grado de obligatoriedad de sus normas, siendo el último más propio de la cultura jurídica anglosajona ${ }^{35}$. A diferencia del hard law, el soft law se integra de una serie de normas no vinculantes que, por lo tanto, no entrañan una obligación jurídica. La doctrina anglosajona expresa que el hard law cuenta con tres elementos esenciales: precisión, obligatoriedad y delegación. Por su parte, en el soft law el segundo elemento se presenta de forma atemperada ${ }^{36}$ y "la observancia de sus preceptos es el resultado de una voluntaria adecuación de sus asignatarios y no corresponde a una verdadera opinio juris ac necessitatis, como sería el caso de la costumbre como fuente del derecho internacional" 37 .

Durante los últimos quince años el desarrollo y la aplicación del soft law ha crecido considerablemente, convirtiéndose en una de las principales herramientas de coordinación jurídica internacional ${ }^{38}$. Esta circunstancia se debe, en gran medida, al fenómeno de la globalización internacional que amplía la calidad y cantidad de relaciones comerciales y humanas al tiempo que acorta los tiempos y las distancias.

El protagonismo del soft law no ha sido ajeno al Derecho tributario internacional, sino todo lo contrario. Tal es así que puede considerarse como uno de los principales instrumentos de coordinación de principios tributarios a escala mundial. El Derecho internacional tributario blando (soft international tax law) incluye cualquier tipo de normas sociales y principios, pero nunca con fuerza obligatoria ${ }^{39}$.

\footnotetext{
35 Robilant, A. (2006). "Genealogies of soft law”. En American Journal of Comparative Law, V. 54, pp. 499 y ss.

36 Аввотt, K. W. \& S Nydal, D., "Hard and soft law in international governance", in 45\% International Organization, 2000, p. 405.

37 Pistone, P., "Tratados fiscales internacionales y soft law" (2008). En El tributo y su aplicación: perspectivas para el siglo XXI. Buenos Aires: Marcial Pons, p. 1197.

38 Pistone, P., (2005) Soft tax coordination: a suitable path for the OECD and the EU to address the challenges of international double (non) taxation in VAT/GST systems, in Lang, Value Added Tax and Direct Taxation-Similarities and Differences. Amsterdam IBFD Publications, p. 101.

39 Pistone (2005) 1165.
} 
Hoy en día los modelos de convenio constituyen la principal expresión del soft international tax law; en especial el MCOCDE y el MCONU. A la hora de confeccionar y renovar sus CDI, los Estados tienen plena libertad para acogerse a las disposiciones de los dichos modelos, los cuales se erigen como meras guías internacionales que pretenden coordinar el contenido de los diferentes acuerdos bilaterales.

En este proceso normativo es interesante destacar que generalmente el soft law precede e inspira al hard law ${ }^{40}$, en el sentido que el soft law acaba transformándose en hard law, pero normalmente no ocurre al revés; de manera forma tal que el primero sirve para la configuración del segundo. El párrafo anterior demuestra claramente este proceso en el Derecho internacional tributario ya que, prima facie, los países pueden adherirse o no a los postulados de los modelos internacionales pero una vez que se han incorporado en los convenios bilaterales su cumplimiento deja de ser voluntario. Sobre esto no cabe ninguna duda.

Mención aparte se merecen los comentarios de los modelos de convenios, en especial los del MCOCDE, ya que el panorama puede llegar a complicarse cuando estamos ante países que no son miembros de dicho organismo. En primer lugar, habrá que definir si tales comentarios constituyen verdaderas normas jurídicas y, dentro de estas, si se trata de soft law o hard law. En segundo término, nos planteamos si la incorporación de una cláusula de los modelos en un CDI implica la adopción automática de sus comentarios.

La primera cuestión no presenta demasiadas complicaciones como la segunda. Si entendemos que los preceptos de los modelos son manifestaciones del soft law, es lógico que sus comentarios ostenten esa misma naturaleza ${ }^{41}$.

La respuesta al segundo problema es más compleja y, a primera vista, podría llegar a depender de la relación de los Estados Contratantes del CDI con la OCDE. En el caso de países que no son miembros de la OCDE parece razonable que los comentarios no afecten directamente las disposiciones adoptadas del MCOCDE. Ello es así porque no han participado directamente en la formulación de las cláusulas ni de los comentarios del modelo, el cual tiene a favorecer lógicamente a sus miembros. Pero si se trata de Estados miembros de dicha organización, como es el caso de Chile, el argumento antes expuesto se desvanece y, tal vez, estaría más justificada una trasposición directa de los comentarios.

Sin embargo, entendemos que incluso los países miembros de la OCDE tampoco deberían quedar automáticamente vinculados por los

\footnotetext{
40 Chinkin, C. M. (2000). Normative development in the international legal system, Oxford: Oxford University Press, p. 30.

41 Engelen, F. (2004) Interpretation of tax treaties under international law. Amsterdam: IBFD Publications, pp. 457-458.
} 
comentarios del MCOCDE. En primer lugar porque, como venimos diciendo, los comentarios también constituyen soft law y, en segundo lugar, porque se podrían generar serios problemas de compatibilidad con el ordenamiento jurídico interno de los diferentes Estados ${ }^{42}$. De manera que aquí estaría el límite del mencionado automatismo.

En definitiva, el soft law no obliga jurídicamente a los Estados pero una vez que es incorporado en la normativa interna pasa a ser hard law y, como tal, adquiere carácter vinculante. Esto es lo que ocurre con los modelos de convenios internacionales cuando sus cláusulas se traspasan a los CDI. Pero los comentarios que interpretan dichas cláusulas no tienen por qué adquirir, de manera automática, la misma condición de hard law, ya sea que se trate de Estados miembros o no de la OCDE.

A partir de ello es evidente que los convenios chilenos, como los de cualquier otro país, no tienen la obligación de adoptar las disposiciones ni los comentarios de ninguno de los modelos de convenio (soft law), pero una vez adoptadas tienen carácter vinculante (hard law). El artículo 7 del MCOCDE 2010 y sus comentarios no son la excepción a esta regla y, por ende, nada impide en principio que Chile prescinda de ellos en sus acuerdos bilaterales.

Teniendo clara la naturaleza jurídica de los preceptos del MCOCDE y su relación con los acuerdos bilaterales, estamos en condiciones de afrontar la segunda cuestión de este punto: definir la postura que viene adoptando Chile en sus convenios frente a las modificaciones del MCOCDE 2010 en cuanto a las utilidades empresariales. Al respecto es preciso hacer dos consideraciones.

En primer lugar, de los CDI existentes al momento de la reforma del artículo 7 del MCOCDE 2010 solo el convenio con Estados Unidos, celebrado en febrero de 2010, fue revisado con posterioridad a dicha reforma. Sin embargo, las revisiones efectuadas al convenio, entre los años 2011 y 2012, no incluyeron las modificaciones de la OCDE ${ }^{43}$. El acuerdo aún no se encuentra vigente.

En segundo lugar, resta por considerar aquellos convenios que fueron celebrados con posterioridad a la reforma del mentado artículo 7 y ver cuál fue su aceptación en aquellos. En este caso fueron solo tres CDI, a saber:

- Austria (aún no vigente): El documento fue firmado el 6 de diciembre de 2012, en Santiago. Por tratarse de un tratado internacional,

42 Pistone (2005) 104 y Pistone (2008) 1201.

43 El convenio fue firmado el 4 de febrero de 2010, en Washington. Por ser un tratado internacional debe ser remitido al Congreso Nacional para cumplir con los trámites constitucionales respectivos. Las partes del convenio han intercambiado notas diplomáticas para modificarlo. La primera modificación se hizo mediante notas intercambiadas el 25 de febrero de 2011 y la última mediante notas de fechas 10 y 21 de febrero de 2012 . 
debe ser remitido al Congreso Nacional para cumplir con los trámites constitucionales respectivos.

- Rusia: El documento fue publicado el 2 de agosto de 2012. El convenio y su Protocolo entraron en vigor internacional el 23 de marzo de 2012 y se aplica con respecto a los impuestos sobre las rentas que se obtengan y a las cantidades que se paguen, abonen en cuenta, se pongan a disposición o se contabilicen como gasto, a partir del 1 de enero del ańo 2013.

- Sudáfrica (aún no vigente): El documento fue firmado el 11 de julio de 2012. Por tratarse de un tratado internacional, debe ser remitido al Congreso Nacional para cumplir con los trámites constitucionales respectivos.

Luego de repasar el articulado de estos tres convenios comprobamos que ninguno adoptó los preceptos del MCOCDE 2010 a los fines de regular los beneficios empresariales. En virtud de ello tenemos como resultado que ninguno de los $28 \mathrm{CDI}$ firmados por Chile sigue, en definitiva, los lineamientos del reformulado artículo 7. Retomando el planteo inicial de este punto podemos decir que el gobierno de Chile decidió no transformar en hard law el nuevo artículo 7 del MCOCDE.

\section{5) CONCLUSIONES}

En el presente trabajo nos propusimos contrastar la nueva versión del mentado artículo con su redacción anterior (MCOCDE 2008) y analizar la repercusión que tuvo, y las que debería tener, la reforma de dicho precepto para los convenios fiscales chilenos. Para ello repasamos brevemente en qué consiste la reforma del artículo 7 del MCOCDE 2010 en comparación con su versión de 2008; analizamos hasta qué punto el MCOCDE es relevante para la configuración de los CDI celebrados por Chile en cuanto a la regulación de los beneficios empresariales y evaluamos, convenio por convenio, las repercusiones concretas que la reforma del artículo 7 del MCOCDE 2010 implicó para la red chilena de CDI.

En primer lugar, si bien se mantuvieron gran parte de las reglas esenciales, es evidente que el artículo 7 del MCOCDE 2010 implicó ciertas modificaciones para su versión de 2008. A continuación señalamos las más importantes:

- Imputación de beneficios a los establecimientos permanentes: se conserva el mismo criterio de atribución de utilidades a los establecimientos permanentes aunque ya no de manera expresa sino a través de sus comentarios.

- Principio de empresa separada e independiente (arm's length principle): se mantiene intacto en la nueva versión solo que esta vez se 
aclara que, al analizar las operaciones realizadas en relación al establecimiento permanente, se han de tener en cuenta: las funciones efectuadas; los activos utilizados y los riesgos asumidos por la casa central.

- Deducción de gastos: el antiguo párrafo 3 del artículo 7 permitía la deducción de los gastos realizados para los fines del establecimiento, incluyendo los gastos de dirección y generales de administración. Los comentarios del nuevo modelo señalan que la deducción de los gastos atańe a la normativa interna de cada Estado Contratante, aunque siempre respectando el principio de empresa separada e independiente (art. 7.2) y de no discriminación (art. 24.3).

- Compra de bienes para la empresa: actualmente, y a diferencia del MCOCDE 2008, ya no es posible excluir de las utilidades del establecimiento aquellas que se derivan de la mera compra de bienes para la empresa.

- Doble imposición: el nuevo artículo 7, en su $3{ }^{\circ}$ párrafo, incorpora un mecanismo de ajuste fiscal para evitar la doble imposición internacional. De todas maneras, su regulación y resultados no varían mucho respecto al artículo 9.2 (idéntico a la versión anterior), con el cual se complementa.

En segundo lugar, en cuanto al modelo de convenio seguido por Chile hemos visto que históricamente el país andino ha seguido los lineamientos de la OCDE a la hora de confeccionar sus CDI, en especial respecto a los beneficios empresariales. A tal punto que solo dos convenios siguen el artículo 7 del MCONU (Canadá y México). En suma, la Administración chilena tiene como norma general adoptar las pautas de la OCDE y como mecanismo excepcional seguir los preceptos de la ONU. Estos resultados no han de extrañarnos ya que la mayoría coincide en situar a Chile más cerca de los países desarrollados que de los Estados en vías de desarrollo, siendo incluso un Estado miembro de la OCDE desde principio de 2010.

Finalmente, hemos comprobado, por un lado, que ninguno de los CDI celebrados con anterioridad al MCOCDE 2010 fue revisado para adaptarse a su nuevo artículo $7 \mathrm{y}$, por el otro, que ninguno de los acuerdos bilaterales celebrados con posterioridad a dicha reforma se acogió a los nuevos postulados de la OCDE.

En definitiva, es evidente que al tratarse de soft law el gobierno chileno no tiene ninguna obligación de adoptar las disposiciones fijadas en el MCOCDE 2010, transformándolas en hard law. No obstante, entendemos que a los fines de mantener una línea coherente con su historia en materia de CDI (siempre conteste con el MCOCDE), sumado a su no muy lejana incorporación como miembro de la OCDE (2010), la Administración chilena debería seguir, al menos en los nuevos tratados, los 
recientes lineamientos de la OCDE. Ante esta elección política va de suyo que tampoco se aplicarán a los convenios existentes los comentarios del nuevo artículo del MCOCDE 2010.

\section{6) Bibliografía}

- 2010 update to the Model Tax Convention, 22th July 2010;

- Аввотт, K. W. \& Snydal, D. (2000). "Hard and soft law in international governance". En $45^{\circ}$ International Organization;

- Cacciapuoti, E. (2010) "I rapporti tra casa madre e stabile organizzazione: tra valore di mercato e costo storico". Rassegna tributaria, No 1, gennaio-febbraio 2010, pp. 198 y ss.;

- Calderón Carrero, J. M. \& Carmona Fernández, N. (2005) Convenios Fiscales Internacionales. Valencia: CISS;

- Calderón Carrero, J. M. (2004): "Comentarios al artículo 7". En Ruiz García, José Ramón \& Calderón Carrero, José Manuel (Coordinadores): Comentarios a los convenios para evitar la doble imposición y prevenir la evasión fiscal concluidos por España (Análisis a la luz del modelo de Convenio de la OCDE y de la legislación y la jurisprudencia española). La Coruña: Fundación Pedro Barrié de la Maza-Instituto de Estudios Económicos de Galicia;

- Castañeda Ricci, S. \& Muñoz García, L. F. (2010) Algunos comentarios en relación con la propuesta de modificación al artículo 7 del Convenio Modelo de la OCDE, en cuanto a la forma de atribuir utilidades a un establecimiento. México: PricewaterhouseCoopers;

- Chinkin, C. M. (2000). Normative development in the international legal system, Oxford: Oxford University Press;

- Cordón Esquerro, T. (Director) (2007) Manual de fiscalidad internacional, Vol. I. Madrid: Instituto de Estudios Fiscales;

- Draft contents of the 2010 update to the Model Tax Convention, 21th May 2010.

- Engelen, F. (2004) Interpretation of tax treaties under international law. Amsterdam: IBFD Publications;

- García Prats, F. A. (1996) El establecimiento permanente. Madrid: Tecnos;

- Pistone, P. (2005) Soft tax coordination: a suitable path for the $O E C D$ and the EU to address the challenges of international double (non) taxation in VAT/GST systems, in Lang, Value Added Tax and Direct Taxation-Similarities and Differences. Amsterdam IBFD Publications;

- Pistone, P., "Tratados fiscales internacionales y soft law" (2008). En El tributo y su aplicación: perspectivas para el siglo XXI. Buenos Aires: Marcial Pons, pp. 1197-1208; 
- Reol Jiménez, T. \& Velayos Jiménez, F. (2007) "Utilidades empresariales con y sin establecimiento permanente". En Cordón Esquerro, T. (Director): Manual de fiscalidad internacional, Vol. I. Madrid: Instituto de Estudios Fiscales;

- Robilant, A. (2006). "Genealogies of soft law". En American Journal of Comparative Law, V. 54, pp. 499 y ss.;

- Ruiz García, J. R. \& Calderón Carrero, J. M. (Coordinadores) (2004) Comentarios a los convenios para evitar la doble imposición y prevenir la evasión fiscal concluidos por España (Análisis a la luz del modelo de Convenio de la OCDE y de la legislación y la jurisprudencia española). La Coruña: Fundación Pedro Barrié de la Maza-Instituto de Estudios Económicos de Galicia;

- Serrano Antón, F. (Director) (2007) Fiscalidad internacional. Madrid: CEF;

- Tonino, H. \& Cooper, J. (2010) Princing a hypothetical. OECD Observer, No 276-277, diciembre 2009 - enero 2010;

- Valente, P. (2010) "Attribuzione del reddito alla stabile organizzazione: il Rapporto OCSE del 2010”. il Fisco, No 43, 22 de novembre 2010, pp. 1-7000;

- Valente, P. (2010) "Il Modello OCSE di Convenzioe contro le doppie imposizioni. La versione 2010". Il Fisco, No 33, 13 de settembre 2010, pp. 1-5333. 